\title{
On the Prym variety of genus 3 covers of genus 1 curves
}

\author{
Christophe Ritzenthaler and Matthieu Romagny
}

\begin{abstract}
Given a generic degree- 2 cover of a genus 1 curve $D$ by a non-hyperelliptic genus 3 curve $C$ over a field $k$ of characteristic different from 2 , we produce an explicit genus 2 curve $X$ such that $\operatorname{Jac}(C)$ is isogenous to $\operatorname{Jac}(D) \times \operatorname{Jac}(X)$. This construction can be seen as a degenerate case of Bruin's result [8].
\end{abstract}

Keywords. genus 3 curves; plane quartics; Prym variety; singular covers

2010 Mathematics Subject Classification. 14Q05

\section{[Français]}

Titre. Sur la variété de Prym des revêtements de genre 3 des courbes de genre 1

Résumé. Étant donné un revêtement de degré 2 générique d'une courbe $D$ de genre 1 par une courbe non-hyperelliptique $C$ de genre 3 sur un corps $k$ de caractéristique différente de 2 , nous produisons une courbe explicite $X$ de genre 2 dont la jacobienne $\operatorname{Jac}(C)$ est isogène à $\operatorname{Jac}(D) \times \operatorname{Jac}(X)$. Cette construction peut être vue comme un cas dégénéré du résultat de Bruin [8].

Received by the Editors on May 18, 2017, and in final form on December 26, 2017. Accepted on March 9, 2018.

Christophe Ritzenthaler

Université de Rennes 1, CNRS, IRMAR - UMR 6625, F-35000, France

e-mail: christophe.ritzenthaler@univ-rennes1.fr

Matthieu Romagny

Université de Rennes 1, CNRS, IRMAR - UMR 6625, F-35000, France

e-mail: matthieu.romagny@univ-rennes1.fr

The first author acknowledges support from the CysMoLog "défi scientifique émergent" of the Université de Rennes 1. 


\section{Contents}

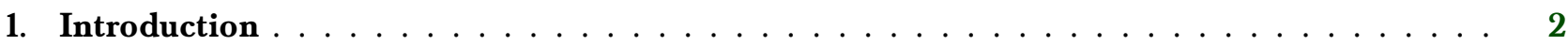

2. From a singular cover to the original cover $\ldots \ldots \ldots \ldots$

3. The singular cover as a special fiber $\ldots \ldots \ldots \ldots \ldots$

\section{Introduction}

Let $C$ be a (smooth, projective, absolutely irreducible) curve of genus $g_{C} \geqslant 2$ over a field $k$. Let us assume that $C$ admits a degree- $n$ map $\pi$ to a curve $D / k$ of genus $g_{D}$ with $0<g_{D}<g_{C}$. We then know that $\operatorname{Jac}(C)$ is isogenous to $\operatorname{Jac}(D) \times A$ and one would like to get as much information as possible on $A$ from $\pi$. For instance, if $C$ is given by explicit equations, is it possible to find a curve $X / k$ given by explicit equations such that $A$ is isogenous over $k$ to $\operatorname{Jac}(X)$ ?

There is an extensive literature dedicated to the case $g_{C}=2$ for which $D$ and $X$ are genus 1 curves, both for its applications to cryptography (see [14], [10] and [4]) and for its interests in pure mathematics [15]. The general theory is well understood (see for instance [20]) and explicit formulas have been worked out when $n=2$ (going back to the work of Jacobi on abelian integrals, see the references in [2, p.395] or [19]), $n=3$ (see [18], [22] or the appendix of [7]), $n=4$ (see [5] and [9]), $n=5$ (see [25]) and more generally when $n \leqslant 11$ (see [23]).

From now on, let $n=2$. When $C$ is given by a hyperelliptic equation, we can describe the map $\pi$ as the quotient by the involution $(x, y) \mapsto(-x, y)$. Then $C: y^{2}=f\left(x^{2}\right)$ where $f$ is a polynomial of degree $g_{C}+1$ and hence $D: y^{2}=f(x)$ is a curve of genus $g_{D}=\left\lceil\frac{g_{C}+1}{2}\right\rceil-1$. One can moreover take advantage of the existence of the hyperelliptic involution to construct the curve $X: y^{2}=x f(x)$ which is the quotient of $C$ by $(x, y) \mapsto(-x,-y)$. It is easy to see by the action on the differentials that $\operatorname{Jac}(C) \sim \operatorname{Jac}(D) \times \operatorname{Jac}(X)$.

Apart from the hyperelliptic cases described above, for a generic curve $C$, a curve $X$ has been made explicit only in few cases. One is when $D$ is hyperelliptic and $C$ is a degree- 2 cover either étale or ramified at two points, see respectively [26, p.346] and [24]. In [8], one can find the only case where $D$ is neither elliptic nor hyperelliptic, namely $g_{C}=5$ and $g_{D}=3$. This work relies on beautiful geometric constructions sketched in [1, VI exercise F]. We will use this construction to get our result.

The present article deals with the case where $C$ is a non-hyperelliptic curve of genus 3 with a degree-2 map to a genus 1 curve $D$ over a field $k$ of characteristic different from 2 . It finds its motivation in the article [28] where cohomological techniques are derived to compute the Weil polynomial of $C$. Although the techniques developed there remain interesting on their own, one byproduct of the present article is that the computation of the Weil polynomial of $C$ can be reduced to computations in genus 1 and 2.

Note that any degree- 2 cover of a genus 1 curve by a non-hyperelliptic genus 3 curve $C$ can be written in the form $y^{4}-h(x, z) y^{2}+r(x, z)=0$ with $r$ a degree 4 polynomial, since we can assume that the involution of $C$ which defines the cover is given by $(x: y: z) \mapsto(x:-y: z)$. Our main result gives an equation of $X$ when $r$ splits as a product of degree 2 factors.

Theorem 1.1. Let $C$ be a smooth, non-hyperelliptic genus 3 curve defined by

$$
C: y^{4}-h(x, z) y^{2}+f(x, z) g(x, z)=0
$$

in $\mathbf{P}^{2}$ where

$$
f=f_{2} x^{2}+f_{1} x z+f_{0} z^{2}, \quad g=g_{2} x^{2}+g_{1} x z+g_{0} z^{2}, \quad h=h_{2} x^{2}+h_{1} x z+h_{0} z^{2}
$$

are homogeneous degree-2 polynomials over a field $k$ of characteristic different from 2. The involution $(x: y$ : $z) \mapsto(x:-y: z)$ induces a degree- 2 cover $\pi$ to the genus 1 curve

$$
D: y^{2}-h(x, z) y+f(x, z) g(x, z)=0
$$


in the weighted projective space $\mathbf{P}(1,2,1)$. Let

$$
A=\left[\begin{array}{lll}
f_{2} & f_{1} & f_{0} \\
h_{2} & h_{1} & h_{0} \\
g_{2} & g_{1} & g_{0}
\end{array}\right]
$$

and assume that $A$ is invertible. Let

$$
A^{-1}=\left[\begin{array}{lll}
a_{1} & b_{1} & c_{1} \\
a_{2} & b_{2} & c_{2} \\
a_{3} & b_{3} & c_{3}
\end{array}\right] .
$$

Then $\operatorname{Jac}(C) \sim \operatorname{Jac}(D) \times \operatorname{Jac}(X)$ with $X \simeq y^{2}=b \cdot\left(b^{2}-a c\right)$ in $\mathbf{P}(1,3,1)$ where

$$
a=a_{1}+2 a_{2} x+a_{3} x^{2}, \quad b=b_{1}+2 b_{2} x+b_{3} x^{2}, \quad c=c_{1}+2 c_{2} x+c_{3} x^{2} .
$$

Remark 1.2. The geometric hypothesis (i.e. which is still true over $\bar{k}$ ) that $\operatorname{det}(A) \neq 0$ is satisfied if $C$ has automorphism group isomorphic to $\mathbf{Z} / 2 \mathbf{Z}$. Indeed since $C$ is non-singular over $\bar{k}$, we can assume that $f=x z, g=x^{2}+g_{1} x z+z^{2}$ ( $f g$ has no double root). If $A$ is non-invertible, we have for instance that $h=\lambda f+\mu g$ but then the morphism $(x: y: z) \mapsto(z: y: x)$ is also an involution. In that case, the Jacobian is going to decompose further in a product of three genus 1 curves which are easily determined.

Remark 1.3. The arithmetic hypothesis that $r=f g$ over $k$ is however necessary in our proof and we do not know how to remove it. Note that it can however always be obtained over a finite extension of $k$.

After we wrote this article, it was brought to our attention by Victor Enolski and Yuri Fedorov that such a case was already handled by Kötter in 1892 (see [21] and also [13, Eq.(2-11)]) in an analytic setting. The proof we propose here is completely different as it is algebraic and works over any field (of characteristic not 2). The shape of the equation we get for $X$ is also much simpler and in particular does not involve any field extension of $k$.

The general strategy is somehow new in this context although it will probably appear very classical to algebraic geometers. The idea is to move from the smooth cover $\pi: C \rightarrow D$ to a singular one $\tilde{\pi}: \tilde{C} \rightarrow \tilde{D}$ of arithmetic genus 5 and 3, with $\tilde{C}$ birational to $C$. The reason to do so is the following. As $n=2$, one can look at $A$ as the Prym variety $P(C / D)$, i.e. the connected component of the identity of $\operatorname{ker}\left(\pi_{*}\right.$ : $\operatorname{Jac}(C) \rightarrow \operatorname{Jac}(D))$. Now, the Prym variety of a degree- 2 cover $\pi$ naturally inherits a principal polarization from $\operatorname{Jac}(C)$ when $\pi$ is étale (see for instance [26, Cor.2]). Our original smooth cover $\pi$ is not étale but the cover $\tilde{\pi}$ will only be ramified over the singular points of $\tilde{D}$. In this case (more precisely in the case of allowable covers, see below), Beauville showed that the generalized Prym variety of $\tilde{\pi}$ is an abelian surface $P(\tilde{C} / \tilde{D})$, isogenous to $P(C / D)$, and naturally principally polarized. The abelian variety $P(\tilde{C} / \tilde{D})$ is therefore the Jacobian of a (possibly reducible) genus 2 curve $X$. It then remains to find $X$ explicitly. In order to do so, we look at the cover $\tilde{C} \rightarrow \tilde{D}$ as the degeneration of a family of unramified degree-2 covers $\mathcal{C} \rightarrow \mathcal{D}$ of smooth genus 3 curves by smooth degree 5 curves. As we said, for the smooth generic fiber, Bruin [8] gives explicitly the hyperelliptic curve $\mathcal{X}$ whose Jacobian is the Prym of the cover. Using a particular deformation, we show that we can specialize the equation of $\mathcal{X}$ to find $X$.

\section{Acknowledgements}

We want to thank Jeroen Sijsling for his comments on an earlier version of the paper and Abhinav Kumar, Victor Enolski and Yuri Fedorov for their references on the subject. 


\section{From a singular cover to the original cover}

Let the notation and hypotheses be as in Theorem 1.1 and let us consider the curve in $\mathrm{P}^{4}$ defined by

$$
\tilde{C}: \begin{cases}x_{1} x_{2} & =f(u, v) \\ x_{2}^{2}+x_{1} x_{3} & =h(u, v) \\ x_{2} x_{3} & =g(u, v)\end{cases}
$$

Multiplying the second equation by $x_{2}^{2}$ and substituting the first and last equation we get our initial curve

$$
C: x_{2}^{4}-h(u, v) x_{2}^{2}+f(u, v) g(u, v)=0 .
$$

Hence we get an isomorphism between $C$ and $\tilde{C}$ on the locus $x_{2} \neq 0$.

Now let us rewrite $\tilde{C}$ using the matrix $A$. We have

$$
\tilde{C}: \operatorname{Id} \cdot\left[\begin{array}{c}
x_{1} x_{2} \\
x_{2}^{2}+x_{1} x_{3} \\
x_{2} x_{3}
\end{array}\right]=A \cdot\left[\begin{array}{c}
u^{2} \\
u v \\
v^{2}
\end{array}\right] .
$$

As $A$ is invertible, we get:

$$
\tilde{C}: A^{-1}\left[\begin{array}{c}
x_{1} x_{2} \\
x_{2}^{2}+x_{1} x_{3} \\
x_{2} x_{3}
\end{array}\right]=\left[\begin{array}{c}
u^{2} \\
u v \\
v^{2}
\end{array}\right]
$$

Let $\left(q_{1}\left(x_{1}, x_{2}, x_{3}\right), q_{2}\left(x_{1}, x_{2}, x_{3}\right), q_{3}\left(x_{1}, x_{2}, x_{3}\right)\right)$ be the left-hand side vector, we get that

$$
\tilde{C}: \begin{cases}q_{1} & =u^{2} \\ q_{2} & =u v \\ q_{3} & =v^{2}\end{cases}
$$

is a degree 2 cover $\tilde{\pi}$ of the curve $\tilde{D}: q_{2}^{2}=q_{1} q_{3}$. In order to see that the covers $\pi$ and $\tilde{\pi}$ are birationally equivalent, let us consider the automorphism $\left(x_{2}: u: v\right) \mapsto\left(-x_{2}: u: v\right)$ of $C$ which induces the cover $\pi$ : $C \rightarrow D$. On $\tilde{C}$, this automorphism induces the automorphism $\left(x_{1}: x_{2}: x_{3}: u: v\right) \mapsto\left(-x_{1}:-x_{2}:-x_{3}: u: v\right)$ since $x_{1} x_{2}=f(u, v)$ and $x_{2} x_{3}=g(u, v)$. But $\left(-x_{1}:-x_{2}:-x_{3}: u: v\right)=\left(x_{1}: x_{2}: x_{3}:-u:-v\right)$ and hence the automorphism is $\tilde{\imath}$ which induces the cover $\tilde{\pi}: \tilde{C} \rightarrow \tilde{D}$. In particular $D$ is birationally equivalent to $\tilde{D}$ over $k$.

To conclude, let us study the singular points of $\tilde{C}$. Since $C$ is non-singular, they can appear only when $x_{2}=0$. But from the first form of the equations of $\tilde{C}$, we see that $f(u, v)=g(u, v)=0$. Since $C$ is non-singular, $f$ and $g$ have no common root, hence $u=v=0$. The possible singular points are then only the points $p_{0}=(0: 0: 1: 0: 0)$ and $p_{\infty}=(1: 0: 0: 0: 0)$. The tangent cone at the point $p_{0}$ has equations $x_{1}=x_{2}=f(u, v)=0$ while the tangent cone at the point $p_{\infty}$ has equations $x_{2}=x_{3}=g(u, v)=0$ (see for instance [27, III, § 3] or [11, p.485]). Since $f$ and $g$ have no double root, it follows that these points are nodal singularities. Therefore we see that :

$(*)$ The fixed points of the involution $\tilde{\iota}$ are exactly the singular points, which are nodal, and $\tilde{\iota}$ preserves the branches at these points.

This condition is phrased in Beauville [3, p.157]. Since the map $\tilde{\pi}$ is ramified at a point $p=(a: b: c:$ $d: e)$ if and only if $\tilde{l}(p)=p$, i.e. if and only if $d=e=0$ and $a b=0, b c=0, b^{2}+a c=0$, we see moreover that $\tilde{\pi}$ is ramified only at the points $p_{0}$ and $p_{\infty}$, in other words:

$(* *)$ The cover $\tilde{\pi}$ is unramified away from the singular locus.

In Donagi and Livné [12], covers satisfying conditions (*) and (**) are called allowable (also admissible by other authors) and we keep this terminology. Finally we sum up the preceding discussion. 
Proposition 2.1. The cover $\tilde{\pi}: \tilde{C} \rightarrow \tilde{D}$ is birationally equivalent to the cover $\pi: C \rightarrow D$ and is allowable.

Let us associate to the cover $\tilde{C} \rightarrow \tilde{D}$ the connected component containing 0 of the kernel of the norm map $\tilde{\pi}_{*}: \operatorname{Jac}(\tilde{C}) \rightarrow \operatorname{Jac}(\tilde{D})$ between generalized Jacobians. We denote it $P(\tilde{C} / \tilde{D})$ and call it the generalized Prym variety. Since the cover $\tilde{C} \rightarrow \tilde{D}$ is allowable, the results [3, Prop.3.5] and [12, Lem.1] show that $P(\tilde{C} / \tilde{D})$ is an abelian variety which is isogenous to the classical Prym variety $P(C / D)$ of the ramified cover $C \rightarrow D$. Moreover the kernel of the isogeny is contained in the group generated by degree 0 divisors supported on the singular points of $\tilde{C}$ and is therefore defined over $k$. We hence get:

Lemma 2.2. The Jacobian of $C$ is isogenous over $k$ to $D \times P(\tilde{C} / \tilde{D})$.

As mentioned in the introduction, what we have gained by moving from $P(C / D)$ to $P(\tilde{C} / \tilde{D})$ is that the latter is principally polarized [3, Th.3.7]. From Weil (see the version in [17, Thm. 3.1]), one knows that every principally polarized abelian surface is either the Jacobian of a curve, the product of two elliptic curves (with the product polarization) or the restriction of scalars of an elliptic curve over a quadratic extension of $k$. Under our hypotheses, we are now going to recover $P(\tilde{C} / \tilde{D})$ as the Jacobian of a genus 2 curve $X$.

\section{The singular cover as a special fiber}

In order to find explicitly the curve $X$, we will 'embed' our singular cover $\tilde{C} \rightarrow \tilde{D}$ into a flat family whose generic fibre is non-singular and use the beautiful result of [8] which we recall here.

Proposition 3.1. Let $Y \rightarrow Z$ be an unramified double cover of a genus 5 curve over a non-hyperelliptic curve of genus 3 over a field $k$ of characteristic different from 2 . Then such a cover can be written $Z: Q_{1} Q_{3}=Q_{2}^{2}$ where $Q_{i}(i=1,2,3)$ are quadratic forms, and

$$
Y:\left\{\begin{array}{l}
Q_{1}=u^{2} \\
Q_{2}=u v . \\
Q_{3}=v^{2}
\end{array}\right.
$$

Moreover the Prym variety $\operatorname{Prym}(Y / Z)$ (as a principally polarized abelian surface over $k$ ) is the Jacobian of the hyperelliptic curve $H$ which is the projective closure of the curve with equation $y^{2}=-\operatorname{det}\left(Q_{1}+2 x Q_{2}+x^{2} Q_{3}\right)$, where we identify $Q_{i}$ with the symmetric $3 \times 3$ matrices $\left(\left(\partial^{2} Q_{i} / \partial x_{j} \partial x_{k}\right)\right)_{1 \leqslant j, k \leqslant 3}$.

Remark 3.2. Bruin's result is stated in characteristic 0 only. But all his arguments work as well in positive characteristic different from 2.

The discriminant $\operatorname{Disc}(F)$ of a degree 4 homogeneous polynomial $F\left(x_{1}, x_{2}, x_{3}\right)$ over a field $K$ of characteristic different from 2 is defined as the multivariate resultant of the partial derivatives of $F$ (see [16, p.426]). It is a polynomial of degree 27 in the coefficients of a generic quartic and $\operatorname{Disc}(F)=0$ if and only if the curve defined by $F$ is singular [16, Chap.13.1.D].

If we restrict the discriminant to quartics $F$ of the form $Q_{2}^{2}-Q_{1} Q_{3}$ where

$$
Q_{i}=\sum_{i_{1}+i_{2}+i_{3}=2} a_{i_{1} i_{2} i_{3}}^{(i)} x_{1}^{i_{1}} x_{2}^{i_{2}} x_{3}^{i_{3}}
$$

are degree 2 homogeneous polynomials over $K$, we get a homogeneous polynomial $H$ of degree $2 \cdot 27=54$ in the coefficients of the $Q_{i}$. Note that $H$ is nonzero since for instance the quartic

$$
x_{1}^{4}-x_{2}^{4}+x_{3}^{4}=\left(x_{1}^{2}\right)^{2}-\left(x_{2}^{2}+x_{3}^{2}\right) \cdot\left(x_{2}^{2}-x_{3}^{2}\right)
$$

has a discriminant equal to $-2^{40}$. The equation $H$ defines a hypersurface in

$$
V=\operatorname{Spec} K\left[a_{i_{1} i_{2} i_{3}}^{(i)}\right] \simeq \mathbb{A}^{18} .
$$


The point $x_{\tilde{D}}$ representing the singular quartic $\tilde{D}=q_{2}^{2}-q_{1} q_{3}$ belongs to $H$ and the pencil

$$
\left(Q_{1}(\lambda), Q_{2}(\lambda), Q_{3}(\lambda)\right)=\left(q_{1}+\lambda q_{1}^{\prime}, q_{2}+\lambda q_{2}^{\prime}, q_{3}+\lambda q_{3}^{\prime}\right), \quad \lambda \in \mathbb{A}^{1}
$$

such that

$$
q_{1}^{\prime}=\left(x_{2}^{2}+x_{3}^{2}\right)-q_{1}, \quad q_{2}^{\prime}=x_{1}^{2}-q_{2}, \quad q_{3}^{\prime}=\left(x_{2}^{2}-x_{3}^{2}\right)-q_{3}
$$

is not contained in $H$ so its generic element is smooth.

Now, let $\mathcal{S}$ be the spectrum of the discrete valuation ring $R=k[[\epsilon]]$ with special fiber $k$ and generic fiber $K$. Over $\mathcal{S}$ we can define a cover $\pi^{\text {def }}: \mathcal{C} \rightarrow \mathcal{D}$ by

$$
\mathcal{C}:\left\{\begin{array}{l}
Q_{1}(\epsilon)=u^{2} \\
Q_{2}(\epsilon)=u v \rightarrow \mathcal{D}: Q_{2}(\epsilon)^{2}-Q_{1}(\epsilon) Q_{3}(\epsilon)=0 . \\
Q_{3}(\epsilon)=v^{2}
\end{array}\right.
$$

Clearly $\pi^{\text {def }}$ is a deformation of $\tilde{\pi}: \tilde{C} \rightarrow \tilde{D}$ and by construction its generic fiber $\pi_{K}: \mathcal{C}_{K} \rightarrow \mathcal{D}_{K}$ is smooth over $K$. The Prym varieties of the fibres of $\pi^{\text {def }}$ fit in a flat family which we call the Prym scheme $\mathcal{P}=P(\mathcal{C} \rightarrow$ $\mathcal{D})$. The details of its construction are to be found in [3], Section 6, especially (6.2); we recall them briefly. The morphism $\pi_{*}^{\text {def }}: \operatorname{Pic}^{0}(\mathcal{C}) \rightarrow \operatorname{Pic}^{0}(\mathcal{D})$ is surjective with smooth target hence it is flat, and its fibres are smooth. Therefore the group scheme $\operatorname{ker}\left(\pi_{*}^{\text {def }}\right) \rightarrow \mathcal{S}$ is smooth and we let $\mathcal{P} \rightarrow \mathcal{S}$ denote the connected component of the unit section. This is the Prym scheme; it has proper fibres hence it is an abelian scheme. Using Proposition 3.1 as well as the fact that the functor $\mathrm{Pic}^{0}$ commutes with base change, we get the following lemma.

Lemma 3.3. The special fiber of $\mathcal{P}$ is $P(\tilde{C} / \tilde{D})$ and the generic fiber is $P\left(\mathcal{C}_{K} / \mathcal{D}_{K}\right)=\operatorname{Jac}\left(X_{K}\right)$ where $X_{K}: y^{2}=$ $-\operatorname{det}\left(Q_{1}(\epsilon)+2 x Q_{2}(\epsilon)+x^{2} Q_{3}(\epsilon)\right)$.

Let $\mathcal{X} / \mathcal{S}$ be the projective closure of $\operatorname{Spec}\left(R[x, y] /\left(y^{2}+\operatorname{det}\left(Q_{1}(\epsilon)+2 x Q_{2}(\epsilon)+x^{2} Q_{3}(\epsilon)\right)\right.\right.$ in the weighted projective space $\mathbf{P}^{(1,3,1)}$, in other words the projective hypersurface described by the homogenized equation. The special fiber $X=\mathcal{X} \otimes k$ is the completion of

$$
y^{2}=-\operatorname{det}\left(Q_{1}(0)+2 x Q_{2}(0)+x^{2} Q_{3}(0)\right)=-\operatorname{det}\left(q_{1}+2 x q_{2}+x^{2} q_{3}\right) .
$$

We are going to prove the following lemma.

Lemma 3.4. The scheme $\mathcal{X}$ is smooth over $\mathcal{S}$ with geometrically connected fibres.

Proof. As we are considering our schemes in the weighted projective space $\mathbf{P}(1,3,1)$, the point at infinity is smooth and we only have to consider the affine part in what follows. Since the generic fiber $X_{K}$ is smooth, we need to consider the singular points of the special fiber $X$. They correspond to points where $F(x)=\operatorname{det}\left(q_{1}+2 x q_{2}+x^{2} q_{3}\right)$ has a (projective) root with multiplicity greater than 1 . With the notation of Theorem 1.1, since

$$
\left[\begin{array}{l}
q_{1} \\
q_{2} \\
q_{3}
\end{array}\right]=\left[\begin{array}{lll}
a_{1} & b_{1} & c_{1} \\
a_{2} & b_{2} & c_{2} \\
a_{3} & b_{3} & c_{3}
\end{array}\right]\left[\begin{array}{c}
x_{1} x_{2} \\
x_{2}^{2}+x_{1} x_{3} \\
x_{2} x_{3}
\end{array}\right]
$$

we have $F=b \cdot\left(b^{2}-a c\right)$. We are actually going to prove that $F$ has no multiple root. As this is a geometric problem, we can assume that we work over an algebraically closed field. In that case, since $C$ is assumed to be non-singular, we can take $f(x, z)=x z$ and $g(x, z)=g_{2} x^{2}+g_{1} x z+z^{2}$. A computation with a computer algebra system such as Magma shows that

$$
\operatorname{Disc}(F)=\frac{4 \cdot g_{2} \cdot\left(g_{2}-g_{1}^{2} / 4\right)^{2} \cdot \operatorname{Disc}\left(h^{2}-4 f g\right)}{\operatorname{det}(A)^{18}} .
$$


Since the curve $D$ is assumed to be non-singular, we see that $4 f g-h^{2}$ has no multiple root, hence $\operatorname{Disc}\left(h^{2}-4 f g\right) \neq 0$. The curve $C$ being non-singular, one has that $g_{2} \neq 0$ (otherwise $(1: 0: 0)$ would be a singular point) and one finds that $g$ does not have a double root, hence $g_{2}-g_{1}^{2} / 4 \neq 0$. We hence get $\operatorname{Disc}(F) \neq 0$.

Finally, since $\mathcal{X} \rightarrow \mathcal{S}$ is smooth and proper, the number of connected components of the fibres is constant on $\mathcal{S}$, hence equal to 1 .

With this result on $\mathcal{X}$, we have the right hypotheses to apply the following proposition (see for instance [6, Th. 9.5/1]).

\section{Proposition 3.5. $\operatorname{Pic}^{0}(\mathcal{X})$ is a Néron model of $\mathrm{Jac}\left(X_{K}\right)$.}

We can now conclude. By Lemma 3.3, the Néron model of the Jacobian of $X_{K}$ is the Néron model of the generic fiber $P\left(\mathcal{C}_{K} / \mathcal{D}_{K}\right)$ of $\mathcal{P}$. Since the special fiber of $\mathcal{P}$ is $P(\tilde{C} / \tilde{D})$ which is an abelian variety (the cover $\tilde{\pi}$ being allowable), we see that the Néron model of $P\left(\mathcal{C}_{K} / \mathcal{D}_{K}\right)$ is $\mathcal{P}$. Hence the Néron model of $\operatorname{Jac}\left(X_{K}\right)$ is $\mathcal{P}$ and by Proposition 3.5 we have $\mathcal{P}=\operatorname{Pic}^{0}(\mathcal{X})$. Taking the special fiber gives:

$$
\mathcal{P} \otimes k=P(\tilde{C} / \tilde{D})=\operatorname{Pic}^{0}(\mathcal{X}) \otimes k=\operatorname{Jac}(\mathcal{X} \otimes k)=\operatorname{Jac}(X) .
$$

We have therefore obtained a description of $P(\tilde{C} / \tilde{D})$ which is isogenous to $P(C / D)$ and this concludes the proof of Theorem 1.1.

\section{References}

[1] E. Arbarello, M. Cornalba, P. Griffiths, and J. Harris, Geometry of algebraic curves, Vol. I, Grundlehren der Mathematischen Wissenschaften, vol. 267, Springer-Verlag, New-York, 1985. MR-0770932

[2] H. F. Baker, Abelian functions. Abel's theorem and the allied theory of theta functions. Reprint of the 1897 original, with a foreword by Igor Krichever. Cambridge Mathematical Library, Cambridge University Press, Cambridge, 1995. MR-1386644

[3] A. Beauville, Prym varieties and the Schottky problem, Invent. Math. 41 (1977), no. 2, 149-196. MR0572974

[4] D. J. Bernstein and T. Lange, Hyper-and-elliptic-curve cryptography, LMS J. Comput. Math. 17 (2014), suppl. A, 181-202. MR-3240803

[5] O. Bolza, Über die Reduction hyperelliptischer Integrale erster Ordnung und erster Gattung auf elliptische durch eine Transformation vierten Grades, Math. Ann. 28 (1887), 447-456. MathDoc

[6] S. Bosch, W. Lütkebohmert, and M. Raynaud, Néron models, Ergebnisse der Mathematik und ihrer Grenzgebiete (3), vol. 21, Springer-Verlag, Berlin, 1990. MR-1045822

[7] R. Bröker, E. W. Howe, K. E. Lauter, and P. Stevenhagen, Genus-2 curves and Jacobians with a given number of points, LMS J. Comput. Math. 18 (2015), no. 1, 170-197. MR-3349314

[8] N. Bruin, The arithmetic of Prym varieties in genus 3, Compos. Math. 144 (2008), no. 2, 317-338. MR2406115

[9] N. Bruin and K. Doerksen, The arithmetic of genus two curves with $(4,4)$-split Jacobians, Canad. J. Math. 63 (2011), no. 5, 992-1024. MR-2866068

[10] R. Cosset, Factorization with genus 2 curves, Math. Comp. 79 (2010), no. 270, 1191-1208. MR-2600562 
[11] D. Cox, J. Little, and D. O'Shea, Ideals, varieties, and algorithms. An introduction to computational algebraic geometry and commutative algebra. Second edition. Undergraduate Texts in Mathematics, Springer-Verlag, New York, 1997. MR-1417938

[12] R. Donagi and R. Livné, The arithmetic-geometric mean and isogenies for curves of higher genus, Ann. Scuola Norm. Sup. Pisa Cl. Sci. (4) 28 (1999), no. 2, 323-339. MR-1736231

[13] V. Z. Enolski and Y. N. Fedorov, Algebraic description of jacobians isogeneous to certain Prym varieties with polarization (1,2), Experimental Mathematics, to appear. arXiv:1411.6143

[14] D. M. Freeman and T. Satoh, Constructing pairing-friendly hyperelliptic curves using Weil restriction, J. of Number Theory 131 (2011), no. 5, 959-983. MR-2772481

[15] G. Frey and E. Kani, Curves of genus 2 covering elliptic curves and an arithmetical application. In: Arithmetic algebraic geometry (Texel, 1989), pp. 153-176, Progr. Math., vol. 89, Birkhäuser Boston, Boston, MA, 1991. MR-1085258

[16] I. M. Gelfand, M. M. Kapranov, and A. V. Zelevinsky, Discriminants, resultants, and multidimensional determinants, Mathematics: Theory \& Applications, Birkhäuser Boston Inc., Boston, MA, 1994. MR1264417

[17] J. González, J. Guàrdia, and V. Rotger, Abelian surfaces of $\mathrm{GL}_{2}$-type as Jacobians of curves, Acta Arith. 116 (2005), no. 3, 263-287. MR-2114780

[18] E. Goursat, Sur la réduction des intégrales hyperelliptiques, Bull. Soc. Math. France 13 (1885), 143-162. MR-1503964

[19] E. W. Howe, F. Leprévost, and B. Poonen, Large torsion subgroups of split Jacobians of curves of genus two or three, Forum Math. 12 (2000), no. 3, 315-364. MR-1748483

[20] E. Kani, The number of curves of genus two with elliptic differentials, J. Reine Angew. Math. 485 (1997), 93-121. MR-1442190

[21] F. Kötter, Ueber die Bewegung eines festen Körpers in einer Flüssigkeit, J. Reine Angew. Math. 109 (1892), 51-81 and 89-111. MR-1580255 and MR-1580257

[22] R. M. Kuhn, Curves of genus 2 with split Jacobian, Trans. Amer. Math. Soc. 307 (1988), no. 1, 41-49. MR-0936803

[23] A. Kumar, Hilbert modular surfaces for square discriminants and elliptic subfields of genus 2 function fields, Res. Math. Sci. 2 (2015), Art. 24, 46 pp. MR-3427148

[24] A. Levin, Siegel's theorem and the Shafarevich conjecture, J. Théor. Nombres Bordeaux 24 (2012), no. 3, 705-727. MR-3010636

[25] K. Magaard, T. Shaska, and H. Völklein, Genus 2 curves that admit a degree 5 map to an elliptic curve, Forum Math. 21 (2009), no. 3, 547-566. MR-2526800

[26] D. Mumford, Prym varieties. I. In: Contributions to analysis (a collection of papers dedicated to Lipman Bers), pp. 325-350. Academic Press, New York, 1974. MR-0379510

[27] D. Mumford, The red book of varieties and schemes. Second, expanded edition. Includes the Michigan lectures (1974) on curves and their Jacobians. With contributions by Enrico Arbarello. Lecture Notes in Mathematics, vol. 1358, Springer-Verlag, Berlin, 1999. MR-1748380

[28] Y.-D. Shieh, Point counting on non-hyperelliptic genus 3 curves with automorphism group $\mathbb{Z} / 2 \mathbb{Z}$ using Monsky-Washnitzer cohomology. In: Algorithmic arithmetic, geometry, and coding theory, pp. 173-189, Contemp. Math., vol. 637, Amer. Math. Soc., Providence, RI, 2015. MR-3364448 